\title{
Printable sensors for explosive detonation
}

\author{
Matthew J. Griffith, ${ }^{1, a)}$ Nathan A. Cooling, ${ }^{1}$ Daniel C. Elkington, ${ }^{1}$ Elmar Muller, ${ }^{2}$ \\ Warwick J. Belcher, ${ }^{1}$ and Paul C. Dastoor ${ }^{1}$ \\ ${ }^{1}$ Priority Research Centre for Organic Electronics, University of Newcastle, University Drive, Callaghan, \\ New South Wales 2308, Australia \\ ${ }^{2}$ AEL Mining Services Ltd., I Platinum Drive, Modderfontein, Johannesburg, 1645, South Africa
}

(Received 18 July 2014; accepted 17 September 2014; published online 6 October 2014)

\begin{abstract}
Here, we report the development of an organic thin film transistor (OTFT) based on printable solution processed polymers and employing a quantum tunnelling composite material as a sensor to convert the pressure wave output from detonation transmission tubing (shock tube) into an inherently amplified electronic signal for explosives initiation. The organic electronic detector allows detection of the signal in a low voltage operating range, an essential feature for sites employing live ordinances that is not provided by conventional electronic devices. We show that a 30 -fold change in detector response is possible using the presented detector assembly. Degradation of the OTFT response with both time and repeated voltage scans was characterised, and device lifetime is shown to be consistent with the requirements for on-site printing and usage. The integration of a low cost organic electronic detector with inexpensive shock tube transmission fuse presents attractive avenues for the development of cheap and simple assemblies for precisely timed initiation of explosive chains. @ 2014 AIP Publishing LLC. [http://dx.doi.org/10.1063/1.4897140]
\end{abstract}

Initiation of explosive chains is an area of great importance to several industries. Currently, the majority of explosive detonator technologies offer initiation through either electronic or pyrotechnic elements. ${ }^{1}$ Electronic detonators offer precise control of explosion timing and the ability to deliver initiation signals to a series of individual explosives. ${ }^{2}$ However, electronic detonators are relatively expensive, require complex wire connections, are prone to triggering from false stimuli such as radio-frequency pick-up and often require substantial power pulses for initiation. ${ }^{3,4}$ Although emerging technology can circumvent some of these issues with wireless initiation signals and radio frequency identification safety mechanisms, such features add significant complexity and cost to the detonation apparatus. ${ }^{5}$ An alternative pyrotechnic initiation method is detonation transmission tubing (shock tube), which consists of hollow polymer tube coated with a layer of high explosive. Upon ignition, a blast wave consisting of several detectable physical stimuli (light, pressure, heat, and gas) propagates down the tube.$^{6-8}$ These stimuli can then initiate a secondary pyrotechnic element to trigger detonation. ${ }^{2,9}$ Although shock tube is a robust, low cost, and easily deployed triggering mechanism, it lacks the control of electronic detonation systems. Thus, a method for interfacing shock tube with electronic timing circuitry would provide both simplicity and control in explosives detonation.

Organic thin film transistors (OTFTs) transduce physical stimuli into electronic signals, and hence offer a pathway to printable low cost sensors for explosive. ${ }^{10}$ Indeed, OTFT arrays have already shown great promise as signal transducers to convert pulsed light ${ }^{11,12}$ and pressure ${ }^{13,14}$ stimuli into an electronic output signal. The devices can be printed at the micrometer scale ${ }^{15,16}$ onto low cost plastic substrates ${ }^{17}$ and hence can be much cheaper than standard inorganic

\footnotetext{
${ }^{\text {a) }}$ Author to whom correspondence should be addressed. Electronic mail: matthew.griffith@newcastle.edu.au
}

electronic devices, a highly desirable feature in a detector unit that will be destroyed upon detonation. The current signal produced by the transistor is inherently amplified and thus readily interfaced with other electronic components. Furthermore, OTFTs can operate at low voltages $(\sim 1-2 \mathrm{~V}),{ }^{18}$ thus, allowing these devices to meet the low power requirements generally observed in explosive environments ${ }^{19}$ and therefore are ideally suited to interfacing shock tube with the electronic circuitry needed to control explosive detonation. ${ }^{20}$

One approach for coupling the shock tube output to an OTFT is to employ a pressure sensitive quantum tunnelling composite (QTC) material, which comprises a low cost rubber matrix into which conducting materials are impregnated. ${ }^{21}$ These materials are utilized in the robotics industry to create pressure sensitive "e-skin." 22 Upon application of pressure to the rubber, increased conductivities are achieved through quantum tunnelling of electrons between the conductive components. ${ }^{23,24}$ Moreover, QTC materials integrated with complex vacuum-processed organic electronic devices have been reported. ${ }^{25}$

A key motivation for this work is to demonstrate the first step towards a low cost organic electronic pressure sensor that can be printed on-site without the need for vacuum processing. In this letter, we report a printable sensor architecture for the detection of the pressure pulse produced by a shock tube blast wave utilising QTC material coupled to an OTFT transducer. The transistor is fabricated using solution processed polymers, which can be readily translated into a fully printable design. We show that this pressure sensor exhibits a highly selective current response to pressure input. Finally, we quantify the degradation of the organic electronic pressure sensor and validate a device shelf-life that is suitable for on-site printing. As such, the work presented here demonstrates the first practical self-contained architecture for a printable pressure sensor for explosives applications. 
Figure 1(a) shows the architecture of the OTFT-based pressure sensor, which consists of pressure sensitive QTC material (purchased from Zoflex (ZL45.1)) coupled with an organic transistor consisting of poly(3-hexylthipohene) as the semiconducting layer, poly(vinylphenol) as the dielectric layer, and poly(3,4-ethylenedioxythiophene) and poly(styrenesulfonate) as the gate electrode. Glass substrates coated with pre-patterned indium-doped tin oxide to produce a source-drain channel $3 \mathrm{~mm}$ by $20 \mu \mathrm{m}$ were purchased from Kintec Company $\left(15 \Omega \square^{-1}\right.$ ). The semiconducting polymer layer was deposited by spin-coating a $20 \mathrm{mg} \mathrm{ml}^{-1}$ chloroform solution onto the substrates at $2000 \mathrm{rpm}$ using a Laurell WS-400A-6NPP/LITE spin-coater. After drying at $45^{\circ} \mathrm{C}$ for 15 min, typical film thicknesses, measured using an Alpha Step 500 profilometer, were $100 \mathrm{~nm}$. The dielectric layer was subsequently deposited by spin-coating an $80 \mathrm{mg} \mathrm{ml}^{-1}$ solution of poly(vinylphenol) in ethanol onto the semiconducting films at $2000 \mathrm{rpm}$. The outer edges of the electrodes were then exposed by removing the polymer layers with an ethyl acetate rinse, and the dielectric layer was dried at $85^{\circ} \mathrm{C}$ for $45 \mathrm{~min}$. Typical dielectric layers were determined to be $400 \mathrm{~nm}$ from profilometry measurements. The gate electrode was subsequently fashioned by drop-casting an aqueous solution of poly(3,4-ethylenedioxythiophene) doped with poly(styrenesulfonate) (HC Starck) onto the dielectric, followed by drying at $45^{\circ} \mathrm{C}$ for $15 \mathrm{~min}$.

The QTC material was coupled to the OTFT through a voltage divider circuit, which controlled the gate voltage of the transistor (Figure 1(b)). The resistance of the QTC material was measured to change from $>1 \mathrm{M} \Omega$ without compression to $<2 \Omega$ under the pressure level associated with shock tube output. Consequently, the divider circuit employed here acts as a switch. Without applied pressure on the QTC sensor, the output voltage from the divider circuit is negligible, whilst under pressure the gate voltage applied to the OTFT is close to that of the input power supply (1.6 V). An integrated detector was fabricated by coupling the shock tube (used as received from AEL Mining Services Ltd. (AEL ZA 291010 02:01 L2)) to the QTC material using in-house constructed testing apparatus (Figure 1(c)). This arrangement avoids damaging the sensor with the high intensity localized pressure pulse from the tubing. The apparatus employed a $60 \mathrm{ml}$ polymeric syringe to act as a piston, which was coupled to the shock tube using a $3 \mathrm{~mm}$ Swagelok fitting. Copper electrodes were fitted square to the QTC edges on each side of the sensor to act as electrodes for connection to the OTFT transducer.

Upon initiation of the shock tube using a commercial tube starter (EIT Corporation, model HR1), the cavity was pressurized and the piston impinged upon the QTC material resulting in a latched pressure state. Figure 1(d) displays the resultant input gate voltage to the OTFT, showing that the piston successfully confines the QTC material into a compressed state, allowing charge to flow through the circuit and a voltage at the gate electrode of $1.25 \mathrm{~V}$ to be observed for at least $40 \mathrm{~s}$. This gate electrode voltage corresponds to a reduction in $\mathrm{V}_{\mathrm{g}}$ of $22 \%$, with subsequent analysis of the divider circuit components revealing the QTC material component exhibits a resistance of $280 \Omega$ under compression in the testing apparatus, indicating an applied pressure of $30-40 \mathrm{kPa}$ if contact resistance between the QTC material and the copper electrodes is neglected (Figure 2). Furthermore, the output voltage is stable, indicating a constant pressure on the QTC material from the compressed piston over this time range. This observation confirms that the connection between the piston and shock tube is pneumatically secure, providing a stable gate voltage over a time period more than sufficient to trigger a response from an OTFT transducer.

Figure 2 displays the current-voltage scans for the same OTFT device connected to the full detector apparatus of the shock tube coupled to a piston and a voltage divider circuit: (a) in the absence of the QTC material, (b) with QTC
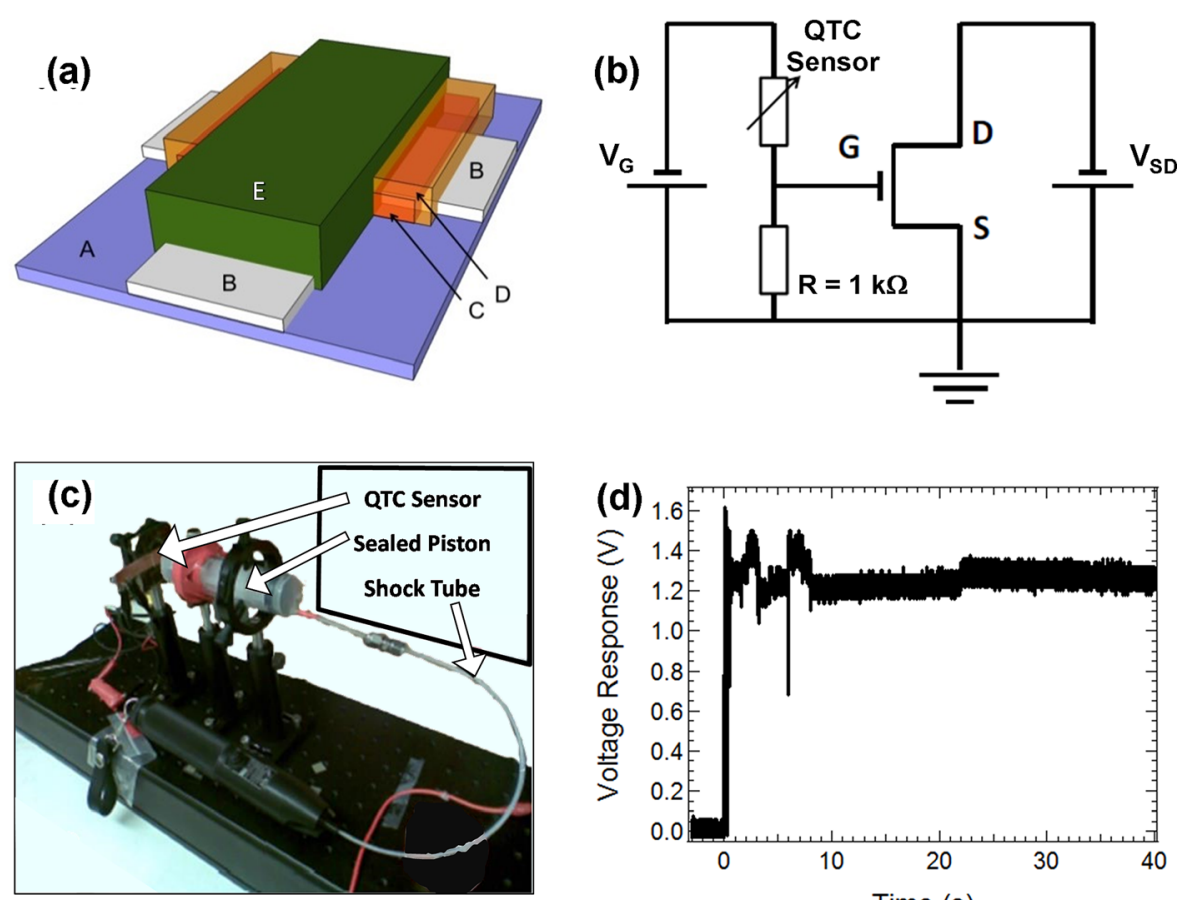

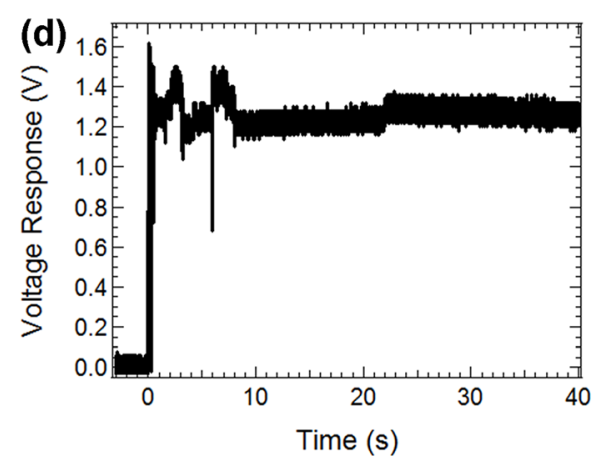

FIG. 1. (a) OTFT device configuration: A: glass, B: indium-doped tin oxide, C: poly(3-hexylthiophene), D: polyvinylphenol, E: poly(3,4-ethylenedioxythiophene)-poly(styrenesul fonate). The QTC material is coupled to E. (b) Circuit diagram of the voltage divider circuit used to control the gate voltage applied to the OTFT. $\mathrm{S}=$ source electrode, $\mathrm{D}=$ drain electrode, $\mathrm{G}=$ gate electrode, and the QTC sensor is represented by the variable resistor. (c) Experimental apparatus used to couple the pressure output from shock tubes to the QTC sensor. (d) Voltage output of the divider circuit upon shock tube initiation and piston compression. 

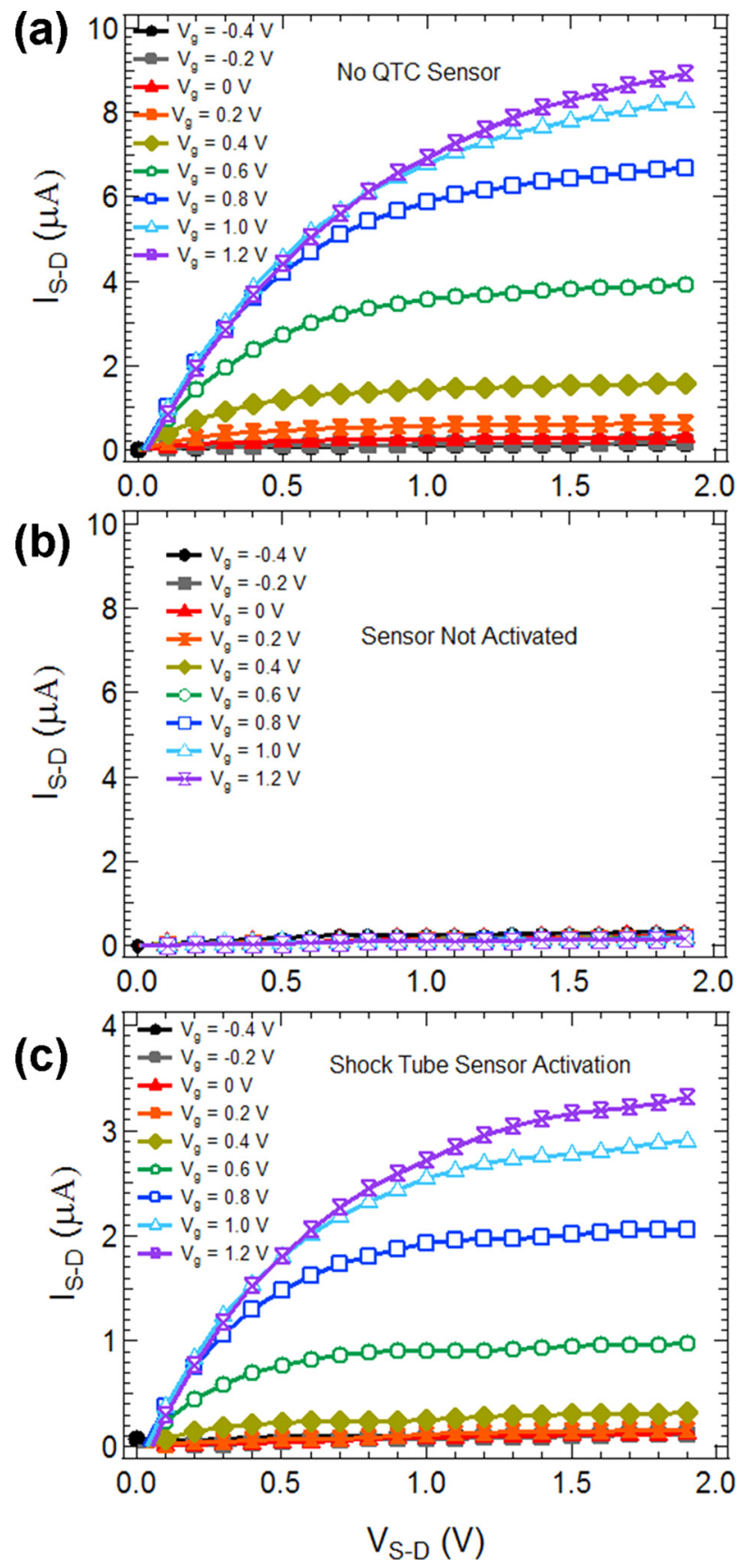

FIG. 2. Current-voltage sweeps under a range of applied gate voltages for: (a) control OTFT device, (b) OTFT device coupled to QTC voltage divider circuit and no shock tube input, and (c) OTFT device coupled to QTC voltage divider circuit with shock tube input.

material but without shock tube activation, and finally (c) with QTC material and subsequent to shock tube initiation into the piston. Figure 2(a) shows that the fabricated OTFTs exhibit classic transistor behaviour as a function of applied gate voltage $\left(\mathrm{V}_{\mathrm{g}}=-0.4 \mathrm{~V}-1.2 \mathrm{~V}\right)$ and indicates that a maximum current of $\sim 9 \mu \mathrm{A}$ can be obtained at the operating voltage of $1.6 \mathrm{~V}$. Upon integration of the QTC material into the device structure and in the absence of the pressure stimulus the OTFT current response is reduced to negligible values, switching the pressure detector into the "off state" for all $\mathrm{V}_{\mathrm{g}}$ (Figure 2(b)). The maximum off-current leakage at $\mathrm{V}_{\mathrm{g}}=1.2 \mathrm{~V}$ reaches $\sim 0.15 \mu \mathrm{A}$, while the highest leakage offcurrent observed was $0.31 \mu \mathrm{A}$ at $\mathrm{V}_{\mathrm{g}}=-0.4 \mathrm{~V}$; indicating the minimum threshold value for a trigger signal from the OTFT transducer. The shock tube was then initiated and the response of the integrated OTFT sensor was recorded (Figure 2(c)). Upon shock tube initiation, the conductivity of the QTC material is increased sufficiently to allow the OTFT response to be again switched between "on" and "off" states by the applied gate voltage. The current-voltage curve after shock tube initiation maintains a high signal to noise ratio, indicating that the conductivity of the QTC material, and hence the voltage at the gate electrode modulated by the QTC material, is stable throughout the duration of the voltage scan. This stability was maintained for all gate voltages tested indicating that the pressure on the QTC material is invariant for the several minutes required to scan this range of gate voltages. The results presented in Figure 2 clearly demonstrate that shock tube output onto the QTC pressure material determines the output of the OTFT, which can then be used as an electronic signal for detonating explosives.

One potential concern for the pressure detector assembly outlined in this study is the influence of degradation on the OTFT output response. Establishing a trigger level for explosives detonation must also account for any reduction in the current signal that occurs due to degradation of the device components with both time and repeated quality control testing procedures. Many explosives components are now fabricated on-site prior to use and thus the OTFT device degradation must be characterised. In particular, the maximum period of time that the shock tube assembly is in contact with the explosive (sleep time) prior to detonation is $48 \mathrm{~h}$. Consequently, the sensor must demonstrate reliable performance even after two days of degradation.

We analysed the degradation of the devices by repeatedly testing the OTFT output under a range of applied gate voltages over $48 \mathrm{~h}$. Following a total of 30 current-voltage sweeps and two days degradation the current response of the OTFT shows a $\sim 36 \%$ reduction compared to that of the freshly fabricated device (Figure 3(a)). Importantly, after degradation, there is still a clear and easily detectable switching behaviour observed in the OTFT-QTC sensor response to pressure output from the shock tube. It was established previously that there is a $\sim 22 \%$ voltage drop across the QTC material under compression due to resistive losses (Figure 1(d)). From the OTFT behaviour shown in Figure 2, this voltage drop results in a corresponding reduction in output current of $\sim 16 \%$ for $\mathrm{V}_{\mathrm{g}}=1.2 \mathrm{~V}$. Consequently, the current output from the degraded OTFT-QTC sensor upon shock tube initiation should be $\sim 50 \%$ of the value measured for a freshly prepared OTFT under the same conditions. To verify the accuracy of this analysis, the output of a freshly prepared OTFT was scaled by $50 \%$ (simulated degradation) to account for degradation loss and compared to the measured output of the OTFT-QTC sensor previously characterised in Figure 2. Since this OTFT-QTC sensor had been subjected to multiple testing cycles and a time delay following fabrication, its current-voltage characteristic corresponds to the output of a degraded device. For applied voltages $>0.5 \mathrm{~V}$, the simulated degradation data show agreement with the measured data to within an error margin of $10 \%$ (Figure 3(b)). As such, the degradation can be factored into calculations of the appropriate trigger level when assembling the integrated pressure detector. Furthermore, the 

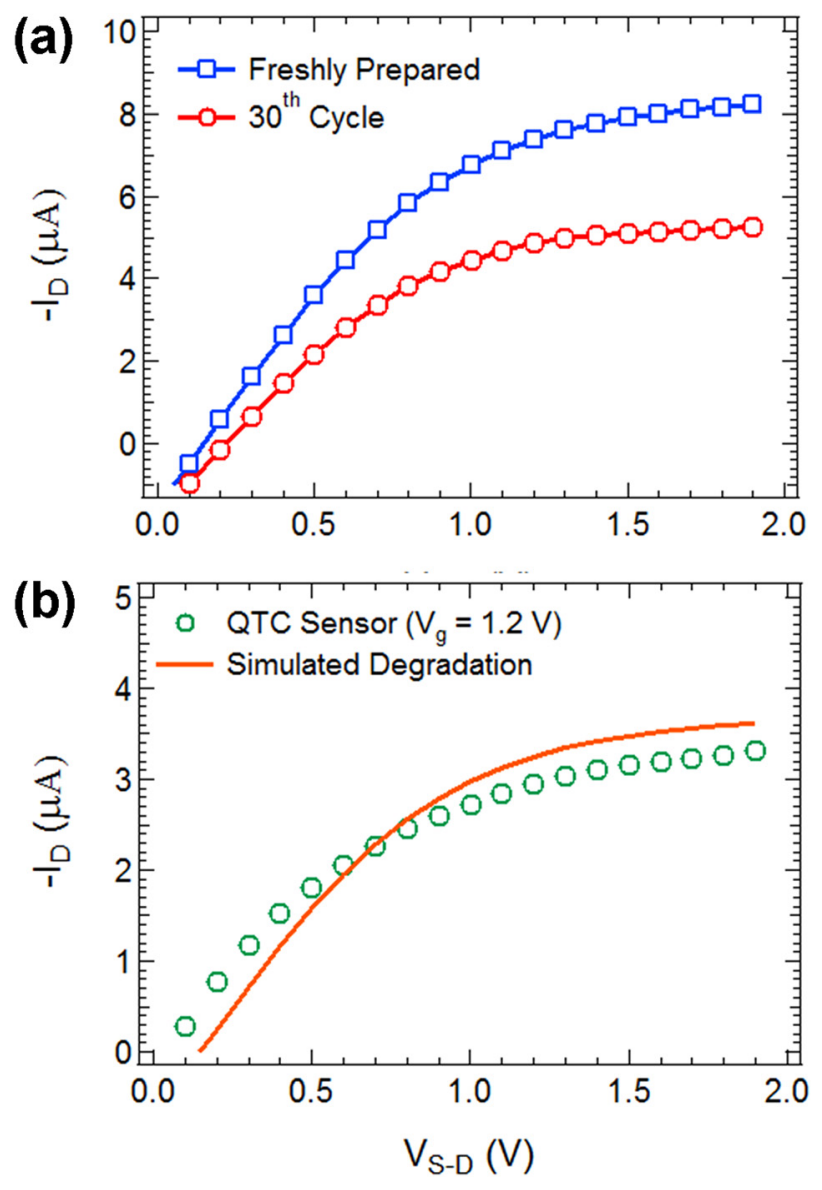

FIG. 3. (a) Initial current-voltage sweep compared to that of the thirtieth testing cycle through a range of gate voltages for the same OTFT device. $\mathrm{V}_{\mathrm{g}}$ for the displayed current-voltage sweeps is $1.2 \mathrm{~V}$. (b) Comparison of the current-voltage sweeps for the integrated OTFT-QTC sensor driving the gate voltage with an input of $1.2 \mathrm{~V}$ after shock tube output activates the sensor, and a simulated OTFT response accounting for degradation from repeated testing cycles and the corrected gate voltage across the device.

demonstration of a 2-day lifetime shows that these devices provide a realistic solution for on-demand printing and usage since this duration is consistent with the maximum allowable sleep time for shock tube assemblies. Finally, the results reported in this study demonstrate that detector assemblies employing a single $1.6 \mathrm{~V}$ battery for power will produce an applied voltage at the gate electrode of the OTFT of $\sim 1.2 \mathrm{~V}$ after accounting for the voltage drop across the QTC material. The current expected from such a device, after accounting for degradation was determined to be $\sim 5 \mu \mathrm{A}$, more than 30 times higher than the current output without any pressure stimuli $(0.16 \mu \mathrm{A})$. Such contrast is more than sufficient to act as a safe trigger signal for the initiation of explosive loadings in practical applications.

In summary, we have described the development of an integrated OTFT-QTC sensor capable of converting the pressure output of shock tube into an electronic trigger signal for explosive detonation. The QTC material was used to moderate the voltage at the gate electrode of an all-polymer printable OTFT. The pressure output from the shock tube, coupled to the sensor through a simple piston arrangement, reduced the resistance of the QTC sufficient to apply a voltage at the gate electrode of $\sim 0.8 \mathrm{~V}_{\mathrm{g}}$. In the absence of any pressure output from the shock tube, the QTC material remained uncompressed and the transistor output was negligible for all values of $\mathrm{V}_{\mathrm{g}}$. The effect of degradation of the OTFT-QTC sensor with both time and repeated testing voltage cycles was quantified within an error of $10 \%$ allowing the reliable determination of on-off switching of the prototype sensor. This work acts as a proof-of-concept for the integration of low cost printable sensors with shock tube for explosives detonation.

The authors thank Dr. Glenn Bryant for his contribution measuring the pressure output from the shock tubes and for valuable discussions. This research was funded through a grant from AEL Mining Services Ltd. This work was performed in part at the Materials node of the Australian National Fabrication Facility, a company established under the National Collaborative Research Infrastructure Strategy to provide nano and microfabrication facilities for Australia's researchers.

${ }^{1}$ M. Cardu, A. Giraudi, and P. Oreste, Rem: Rev. Esc. Minas 66, 375 (2013).

${ }^{2}$ A. S. Babu, K. K. Mishra, P. D. Kshirsagar, H. Shekar, and V. S. Rasane, Def. Sci. J. 63, 305 (2013).

${ }^{3}$ R. Kichouliya, R. Devender, V. V. Ramasarma, D. S. Reddy, and V. G. Borkar, in Proceedings of IEEE International Symposium on Electromagnetic Compatibility (2011), p. 946.

${ }^{4}$ J. J. Pantoja, N. M. Peña, F. Rachidi, F. Vega, and F. Roman, Def. Sci. J. 63, 386 (2013).

${ }^{5}$ P. K. Mishra, M. Bolic, M. C. E. Yagoub, and R. F. Stewart, J. Appl. Geophys. 76, 33 (2012).

${ }^{6}$ D. A. Freiwald, J. Appl. Phys. 43, 2224 (1972).

${ }^{7}$ C. K. Westbrook, Combust. Sci. Technol. 20, 5 (1979).

${ }^{8}$ I. O. Samuelraj, G. Jagadeesh, and K. Kontis, Shock Waves 23, 307 (2013).

${ }^{9}$ P. Zhu, X. Zhou, R.-Q. Shen, Y.-H. Ye, and Y. Han, Chin. J. Energetic Mater. 4, 366 (2011).

${ }^{10}$ J. T. Mabeck and G. G. Malliaras, Anal. Bioanal. Chem. 384, 343 (2006).

${ }^{11}$ G. Yu, Y. Cao, J. Wang, J. McElvain, and A. J. Heeger, Synth. Met. 102, 904 (1999).

${ }^{12}$ J. Labram, P. Wöbkenberg, D. Bradley, and T. Anthopoulos, Org. Electron. 11, 1250 (2010).

${ }^{13}$ I. Manunza and A. Bonfiglio, Biosens. Bioelectron. 22, 2775 (2007).

${ }^{14}$ I. Manunza, A. Sulis, and A. Bonfiglio, Appl. Phys. Lett. 89, 143502 (2006).

${ }^{15}$ T. Sekitani, Y. Noguchi, U. Zschieschang, H. Klauk, and T. Someya, Proc. Natl. Acad. Sci. 105, 4976 (2008).

${ }^{16}$ A. L. Briseno, S. C. B. Mannsfeld, S. A. Jenekhe, Z. Bao, and Y. Xia, Mater. Today 11, 38 (2008).

${ }^{17}$ I. D. W. Samuel, Philos. Trans. R. Soc. London, Ser. A 358, 193 (2000).

${ }^{18}$ D. Elkington, D. Darwis, X. Zhou, W. Belcher, and P. C. Dastoor, Org. Electron. 13, 153 (2012).

${ }^{19}$ R. K. Eckhoff, J. Loss Prev. Process Ind. 15, 305 (2002).

${ }^{20}$ L. Kergoat, B. Piro, M. Berggren, G. Horowitz, and M.-C. Pham, Anal. Bioanal. Chem. 402, 1813 (2012).

${ }^{21}$ T. Someya, T. Sekitani, S. Iba, Y. Kato, H. Kawaguchi, and T. Sakurai, Proc. Natl. Acad. Sci. 101, 9966 (2004).

${ }^{22}$ T. Sekitani and T. Someya, Adv. Mater. 22, $2228(2010)$.

${ }^{23}$ X. Jing, W. Zhao, and L. Lan, J. Mater. Sci. Lett. 19, 377 (2000).

${ }^{24}$ Z. Ounaies, C. Park, K. E. Wise, E. J. Siochi, and J. S. Harrison, Compos. Sci. Technol. 63, 1637 (2003).

${ }^{25}$ Y.-C. Chao, W.-J. Lai, C.-Y. Chen, H.-F. Meng, H.-W. Zan, and S.-F. Horng, Appl. Phys. Lett. 95, 253306 (2009). 